\title{
Tunable laser plasma accelerator based on longitudinal density tailoring
}

\author{
A. J. Gonsalves ${ }^{1}$, K. Nakamura ${ }^{1}$, C. Lin ${ }^{1,2}$, D. Panasenko ${ }^{1 \dagger}$, S. Shiraishi ${ }^{1,3}$, T. Sokollik ${ }^{1,4}$, C. Benedetti ${ }^{1}$, \\ C. B. Schroeder ${ }^{1}$, C. G. R. Geddes ${ }^{1}$, J. van Tilborg ${ }^{1}$, J. Osterhoff ${ }^{1 \dagger}$, E. Esarey ${ }^{1}$, C. Toth ${ }^{1}$ \\ and W. P. Leemans ${ }^{1,4 \star}$
}

\begin{abstract}
Laser plasma accelerators ${ }^{1}$ have produced high-quality electron beams with $\mathrm{GeV}$ energies from $\mathrm{cm}^{-s c a l e}$ devices ${ }^{2}$ and are being investigated as hyperspectral fs light sources producing THz to $\gamma$-ray radiation ${ }^{3-5}$, and as drivers for future highenergy colliders ${ }^{6,7}$. These applications require a high degree of stability, beam quality and tunability. Here we report on a technique to inject electrons into the accelerating field of a laser-driven plasma wave and coupling of this injector to a lower-density, separately tunable plasma for further acceleration. The technique relies on a single laser pulse powering a plasma structure with a tailored longitudinal density profile, to produce beams that can be tuned in the range of $100-400 \mathrm{MeV}$ with per-cent-level stability, using laser pulses of less than $40 \mathrm{TW}$. The resulting device is a simple stand-alone accelerator or the front end for a multistage higher-energy accelerator.
\end{abstract}

Producing high-quality electron beams from an accelerator requires electron injection into the accelerating field to be localized in time and space. For laser plasma accelerators (LPAs) that rely on homogeneous plasmas driven with single laser pulses, continuous injection can occur when driving large-amplitude plasma waves (wakefields), resulting in large energy spread. Lower energy spread can be achieved through termination of injection by operating near the injection threshold or by injecting enough charge to suppress the wake amplitude (that is, beam loading). Subsequent termination of the accelerating process at dephasing (that is, when electrons are starting to outrun the accelerating wave) minimizes energy spread. These mechanisms have produced per-cent-level energy-spread beams ${ }^{2,8-10}$, but small changes in parameters can result in large changes in beam quality. As a result, tunability has been limited, necessitating the development of a simple, robust and controlled injection technique combined with an independently controllable accelerating stage.

In general, injection of electrons into a plasma wave occurs when the velocity of background electrons approaches the wake phase velocity. Laser-based methods for boosting the electron velocity have been proposed ${ }^{11,12}$ and implemented ${ }^{13,14}$ to achieve tunable electron beams, but require sophisticated alignment and synchronization of the multiple laser pulses. Injection can also be triggered by introducing electrons into the correct phase of the wake through ionization ${ }^{15}$, but so far the technique has resulted in broad energy-spread beams with high divergence ${ }^{16,17}$.
A different approach, that relies on a single laser pulse for powering the LPA, is to momentarily slow down the wake phase velocity to facilitate trapping ${ }^{18}$. The control of the wake phase velocity can be achieved by tailoring the nonlinear plasma wavelength $\lambda_{\mathrm{p}}(z)$ along the longitudinal coordinate $z$, through control of the electron density $n_{\mathrm{e}}$ and the laser parameters. Specifically, $\lambda_{\mathrm{p}}(z)=\lambda_{\mathrm{p} 0}(z) F$, where the linear plasma wavelength $\lambda_{\mathrm{p} 0}(\mu \mathrm{m}) \approx 3.3 \times 10^{10} / \sqrt{n_{\mathrm{e}}\left(\mathrm{cm}^{-3}\right)}$ and $F$ is a function of the laser parameters: at low intensity $\left(a_{0}<1\right) F \approx 1$, and when $a_{0} \gtrsim 1$ $F$ can be greater than 1 . Here $a_{0}$ is the normalized laser vector potential $a_{0}=7.3 \times 10^{-19}[\lambda(\mu \mathrm{m})]^{2} I_{0}\left(\mathrm{~W} \mathrm{~cm}^{-2}\right)$, and $I_{0}$ is the peak laser intensity of the laser pulse with wavelength $\lambda$. The wake phase velocity $^{1}$ is $\beta_{\mathrm{p}} \approx \beta_{\mathrm{g}} /\left(1+|\zeta| \lambda_{\mathrm{p}}^{-1} \mathrm{~d} \lambda_{\mathrm{p}} / \mathrm{d} z\right)$, where $\beta_{\mathrm{g}}$ is the laser group velocity and $|\zeta|$ is the distance behind the laser.

Previously, phase-velocity control by using tailored plasmadensity profiles was experimentally achieved for low-energy beams ${ }^{19-21}$. In this paper we demonstrate phase-velocity reduction by combined relativistic intensity and density down-ramp effects in a submillimetre high-density plasma, and the subsequent acceleration of the resulting low-energy bunches $(<30 \mathrm{MeV})$ to several hundred $\mathrm{MeV}$ in a closely coupled lower-density capillary-discharge-based structure. By controlling the densities of the two plasma regions and the focal location of the laser pulse, precise control over trapping and acceleration is achieved and high-quality, stable and tunable electron beams are generated.

Self-focusing can occur when the laser power $P \gtrsim P_{\mathrm{c}}$, where $P_{\mathrm{c}}(G W)=17\left(\lambda_{\mathrm{p} 0} / \lambda\right)^{2} \propto 1 / n_{\mathrm{e}}$ is the critical power for relativistic self-focusing. The high-density plasma can therefore increase both $a_{0}$ and $\mathrm{d} \lambda_{\mathrm{p}} / \mathrm{d} z$, lowering the phase velocity as illustrated in Fig. $1 \mathrm{a}$. After focusing, $\mathrm{d} a_{0} / \mathrm{d} z<0, \mathrm{~d} \lambda_{\mathrm{p}} / \mathrm{d} z<0$, and injection is terminated, resulting in a highly localized injector. The electrons are then accelerated to several hundred $\mathrm{MeV}$ in the integrated plasma channel, powered by the same drive laser pulse, demonstrating post-acceleration similar to that simulated in refs 19,22-24.

The set-up is shown in Fig. 1c. Laser pulses with peak power of $40 \mathrm{TW}$ and pulse length of $38 \mathrm{fs}$ from a Ti:sapphire laser system were focused onto longitudinally tailored plasma targets. The injector stage, a supersonic gas jet, was operated without a capillary over a wide range of parameters (detailed in the Methods section), producing beams with energy up to $60 \mathrm{MeV}$ and energy spread (full-width at half-maximum, FWHM) ranging between $1 \%$ and $50 \%$. A typical electron beam from the injector

\footnotetext{
${ }^{1}$ Lawrence Berkeley National Laboratory, Berkeley, California 94720, USA, ${ }^{2}$ Peking University, Beijing 100871, China, ${ }^{3}$ University of Chicago, Chicago, Illinois 60637, USA, ${ }^{4}$ University of California, Berkeley, California 94720, USA. †Present addresses: Physical Sciences Inc., 6652 Owens Dr., Pleasanton, California 94588, USA (D.P.); University of Hamburg and Deutsches Elektronen-Synchrotron (DESY), D-22607 Hamburg, Germany (J.O.). *e-mail:WPLeemans@lbl.gov.
} 
a

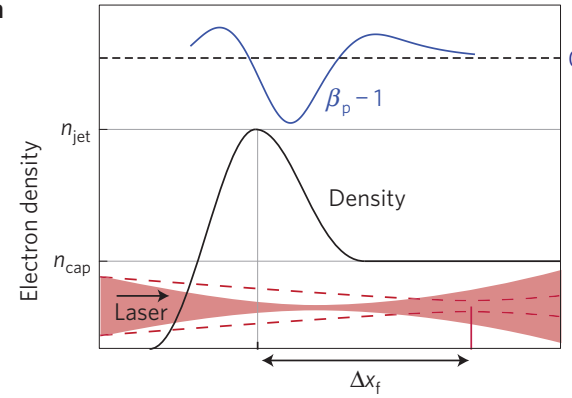

c

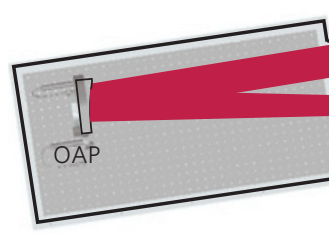

b

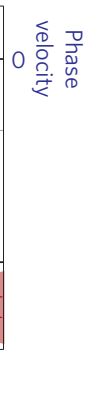

b
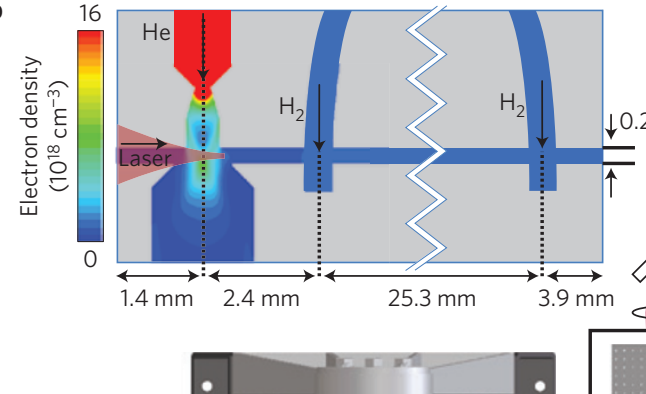
$.22 \mathrm{~mm}$

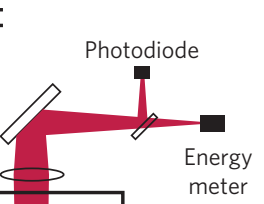

Density-tailored plasma target

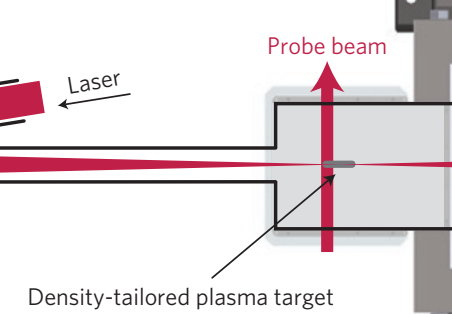

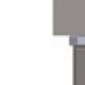

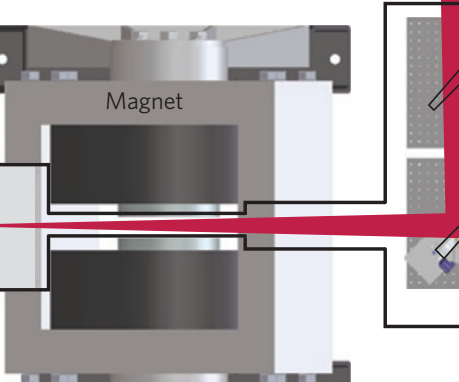
-

Figure 1 | Experimental layout of the density-tailored LPA. a, Illustration of the laser focusing onto the plasma target and of the basic physics: the longitudinal on-axis density profile in the region of the gas (black), the location of the vacuum focus with respect to the centre of the gas jet $\Delta x_{f}$ (red dashed line), the self-focused laser focal position (red shaded area) and the wake phase velocity (blue line). $\mathbf{b}$, The target schematic representation and gas profile calculated by a commercial fluid-dynamics code, where a supersonic gas jet has been embedded into a capillary that is filled with hydrogen gas. c, The experimental layout. Pulses from the LOASIS Ti:sapphire laser system of length $38 \mathrm{fs}$ were focused onto the gas targets to a focal spot size of $r_{0}=22 \mu \mathrm{m}$ using an off-axis parabolic mirror (OAP), producing a peak intensity at focus of $4 \times 10^{18} \mathrm{~W} \mathrm{~cm}^{-2}$. The spectra of electron beams emerging from the plasma were obtained using a magnetic spectrometer ${ }^{29}$, as described in the Methods section. The spectrum and energy of the laser pulses at the capillary output were measured with a spectrometer and pyroelectric energy meter, respectively.

is shown in Fig. 2a, for which the laser energy depletion was $\approx 30 \%$, leaving significant laser energy for driving a wake in a subsequent structure. Electron beams could be produced within the range $0 \mathrm{~mm} \leq \Delta x_{\mathrm{f}} \leq 3.5 \mathrm{~mm}$ and the maximum charge was obtained when focusing $\Delta x_{\mathrm{f}}=2 \mathrm{~mm}$ downstream of the jet centre. Injection for vacuum focus $3.5 \mathrm{~mm}$ downstream of a jet of width $\approx 0.75 \mathrm{~mm}$ is consistent with the focal location shifting closer to the peak of the density profile owing to self-focusing. Phasevelocity reduction due to the increase in laser intensity and the decreasing density on the down-ramp of the gas jet resulted in controlled injection.

The gas jet (injector) was then coupled to a lower-density plasma produced in a capillary-discharge waveguide ${ }^{25-27}$ (accelerator). The geometry is shown in Fig. 1b. The accelerator stage was characterized by not injecting gas into the jet nozzle, such that a homogenous hydrogen plasma was formed between the gas slots. No electrons were detected on the magnetic spectrometer for the density range employed $\left(1 \times 10^{18} \mathrm{~cm}^{-3} \leq n_{\text {cap }} \leq 2.7 \times 10^{18} \mathrm{~cm}^{-3}\right)$, which is consistent with previous experiments ${ }^{2}$. As shown by the shift in the mean of the laser spectral distribution from 803 to $835 \mathrm{~nm}$ (caused by transfer of energy from photons to the wake), a significant wakefield was produced in this darkcurrent-free structure.

When gas was injected into the jet nozzle, high-quality beams were observed with energy significantly higher than that with the jet alone. The increase from tens of $\mathrm{MeV}$ to hundreds of $\mathrm{MeV}$ is consistent with post-acceleration in the lower-density structure. The beam properties were controlled by focus location and by jet density. The effect of focus location is shown in Fig. $2 \mathrm{~b}-\mathrm{f}$, where for $n_{\text {jet }} \approx 7 \times 10^{18} \mathrm{~cm}^{-3}$ and $n_{\text {cap }} \approx 1.8 \times 10^{18} \mathrm{~cm}^{-3}$ each image is an average of 20 consecutive electron beams for various $\Delta x_{\mathrm{f}}$ (compare Fig. 1a). Also shown are lineouts of each image, with the average denoted by the black line and the standard deviation by the red shaded area. The effect of jet density is shown in Fig. $2 \mathrm{~g}$, which shows beam energy (red circles), charge (black squares), and energy spread (triangles). The increased bunch charge at higher density is expected owing to the slower wake phase velocity and increased laser self-focusing and steepening, all of which enhance trapping. For Fig. 2c, which is typical of the performance achieved, the beam energy, energy spread (FWHM) and divergence (FWHM) were $341 \mathrm{MeV}, 11 \%$ and $2.5 \mathrm{mrad}$, respectively. The production of tunable beams with low energy spread and divergence, and with $100 \%$ injection probability (which is not observed for conventional self-trapping), is consistent with realization of phase-velocity triggered injection and post-acceleration as simulated in refs 19,22-24. The average divergence was $2.2 \mathrm{mrad}$ for the consecutive beams in Fig. 2b-f, compared with 5-11 mrad beams produced with other state-of-theart injection techniques ${ }^{14,20}$.

Energy spread was correlated with charge, with a peak observed for $1 \mathrm{pC}$ for Fig. $2 \mathrm{~b}-\mathrm{f}$ and $10 \mathrm{pC}$ for Fig. $2 \mathrm{~g}$. This suggests that beam loading is not increasing the energy spread, but that phasing of injected electrons into the lower-density structure is not optimized. Further evidence for this is that the highest-quality beams had $1 \mathrm{pC}$ charge and energy spread and divergence as low as $1.8 \%$ and $1.4 \mathrm{mrad}$, respectively. For a different parameter regime than the present experiment, electron beams with energy spread less than $1 \%$ have been simulated by post-acceleration after a high-density gas jet ${ }^{19}$. To achieve $1 \%$ energy spread in the simulation, the down-ramp had to be terminated in a plasma channel with uniform axial density immediately after the particles were trapped, such that the electrons were correctly phased in the wake and beam loading was balanced to produce a uniform accelerating field across the bunch. Furthermore, matched guiding was required to prevent further evolution of the laser spot size after injection. Geometrical changes to the design will be made to meet these requirements in future experiments.

Injection of electrons was investigated using the particle-in-cell modality of INF\&RNO (described in the Methods section). Shown in Fig. 3 is the laser vector potential $a_{0}$ (dash-dot line), which 

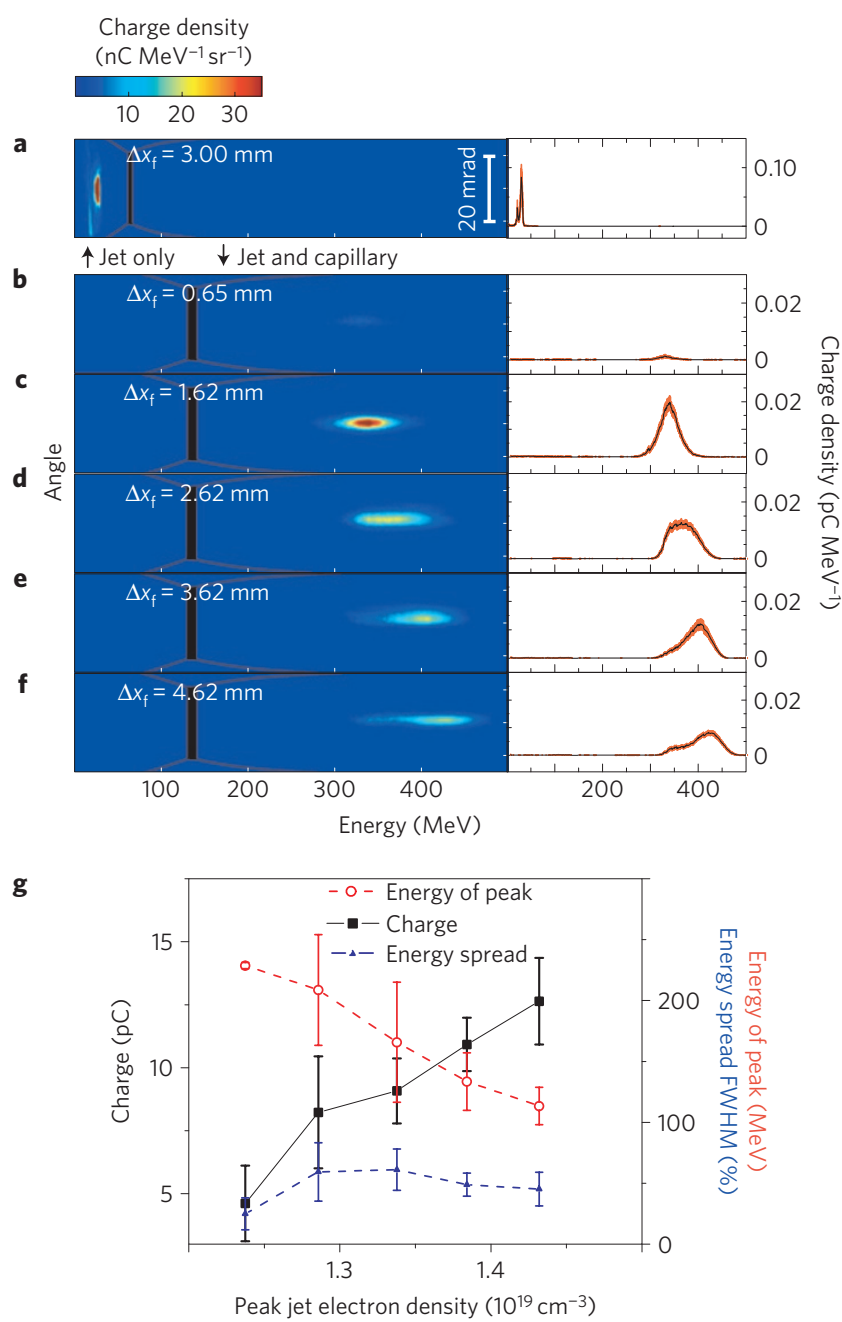

Figure 2 | Electron-beam data from both the gas jet and the density-tailored plasma target, demonstrating the post-injection acceleration in the capillary structure and the control over electron-beam properties afforded by the density-tailored approach. a-f, Averaged magnetic spectrometer images from 20 consecutive shots. The black shaded areas in each image represent the regions not covered by the spectrometer cameras. Lineouts of the mean (black curve) and the standard deviation (red area) are on the right of each image. The LPA in a consisted only of a helium gas jet with peak electron density $n_{\text {jet }} \approx 7 \times 10^{18} \mathrm{~cm}^{-3}$ and length (FWHM) $0.75 \mathrm{~mm}$. For $\mathbf{b}-\mathbf{f}$ a helium gas jet with $n_{\text {jet }} \approx 7 \times 10^{18} \mathrm{~cm}^{-3}$ and length (FWHM) $0.55 \mathrm{~mm}$ was coupled to a capillary with density $n_{\text {cap }} \approx 1.8 \times 10^{18} \mathrm{~cm}^{-3}$ for various focal locations $\Delta x_{\mathrm{f}}$. $\mathbf{g}$, The charge (squares), energy (circles) and energy spread (triangles) as a function of peak jet density for $\Delta x_{f}=0.62 \mathrm{~mm}$ at capillary density $n_{\text {cap }} \approx 1.2 \times 10^{18} \mathrm{~cm}^{-3}$. The error bars correspond to the standard deviation of the data.

increases to $\approx 3.5$, compared with the vacuum focal value of $\approx 1.5$, producing a bubble wakefield. The wake phase velocity (dashed line) is reduced in the region where the density is decreasing and the laser intensity is increasing, and trapping of particles is observed in the grey shaded region. The contribution to the phase-velocity reduction by self-focusing can be determined by comparing the wake phase velocity for a vacuum $a_{0} \approx 1.5$ (dashed line) with a vacuum $a_{0} \approx 0.1$ (red solid line), because for the latter self-focusing can be ignored. It can be seen that self-focusing causes a significant reduction in wake phase velocity. Injection occurs over only a short propagation distance, because the wake phase velocity increases as the ramp is terminated and the laser reaches maximum intensity.

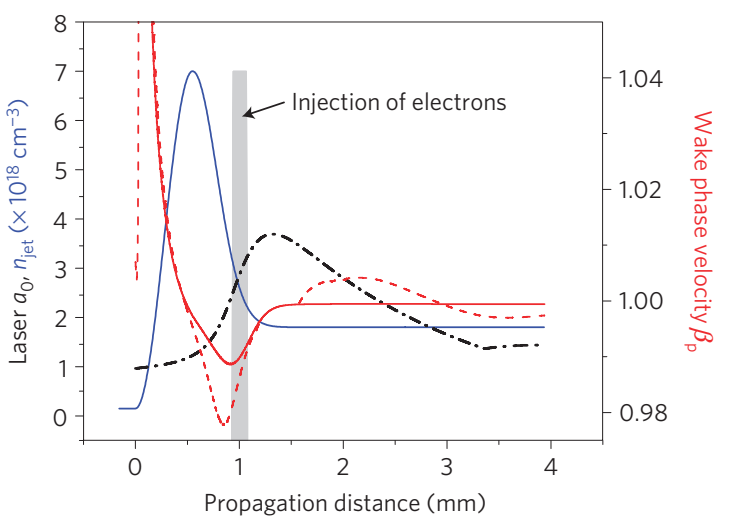

Figure 3 | Simulated evolution of the normalized vector potential $a_{0}$ (dash-dot line) as a function of propagation distance for $\Delta x_{f}=1.6 \mathrm{~mm}$ and $n_{\text {jet }}=7 \times 10^{18} \mathrm{~cm}^{-3}$. The blue solid line shows the longitudinal density profile. The phase-velocity reduction (red dashed line) was larger than that for the down-ramp alone (red solid line) owing to self-focusing increasing the nonlinear plasma wavelength, and trapping of electrons occurred in the grey shaded area. The phase velocities shown are measured in the centre of the bubble behind the laser pulse, where the longitudinal electric field is zero. The phase-velocity reduction at the back of the bubble is approximately a factor of two larger.

The trends observed with $\Delta x_{\mathrm{f}}$ and $n_{\text {jet }}$ were investigated using the fluid modality of INF\&RNO. Figure 4 a shows the effect of the gas jet on the laser intensity and focal position as a function of gas-jet density and laser vacuum focus. The jet causes laser pulse self-focusing, which is increased for lower $\Delta x_{\mathrm{f}}$ or higher $n_{\text {jet }}$. This explains the increase in charge with $n_{\text {jet }}$ observed in Fig. $2 \mathrm{~g}$, because the laser intensity and wakefield amplitude increase, and the injection location is shifted upstream closer to the peak of the density profile. The lower charge observed in Fig. 2b-f can also be understood, because $n_{\text {jet }}$ was approximately a factor of two lower. Further evidence for self-focusing is shown in Fig. 5, where experimental laser spectra are shown for targets of no plasma, jet only and jet with capillary for peak densities in the jet between $4 \times 10^{18}$ and $5.5 \times 10^{18} \mathrm{~cm}^{-3}$. Significantly more redshifting was observed when the capillary was introduced, compared with operating only the jet, showing that a significant wake was present in the capillary. Furthermore, redshifting increased with jet density, consistent with increased laser intensity and a larger wake in the capillary. A similar trend was observed in simulations.

Self-focusing was also the dominant effect in determining energy gain, because increased self-focusing, whether it be from reducing $\Delta x_{\mathrm{f}}$ or increasing $n_{\mathrm{jet}}$, reduces the focused laser spot size and makes diffraction of the laser pulse in the non-matched plasma channel more severe. This reduced the average laser intensity over the channel length and the final energy gain. The energy gain can be estimated by considering the field excited by the focused laser pulse integrated over the interaction length limited by laser diffraction, which is longer than the Rayleigh range owing to nonlinear self-focusing and plasma channel guiding. Figure $4 \mathrm{~b}$ shows the energy gain $m_{\mathrm{e}} c^{2} \Delta \gamma$ for several $\Delta x_{\mathrm{f}}$ calculated using $\Delta \gamma \simeq \pi^{2}\left[\left(a_{0}^{2} / 2\right) / \sqrt{1+a_{0}^{2} / 2}\right]\left(Z_{\text {eff }} / \lambda_{\mathrm{p} 0}\right)$ (ref. 1). Both the laser vector potential at focus $a_{0}$ and the effective acceleration length $Z_{\text {eff }}$ were obtained from simulation. Figure $4 \mathrm{~b}$ shows qualitative agreement between the estimated diffraction-limited energy gain and the experimental measurement shown in Fig. 2. This demonstrates that the key mechanism determining the energy gain is diffraction of the laser pulse, which can be controlled by changing injector and channel parameters. This is in contrast to previous LPA experiments employing the hydrogen-filled 
a

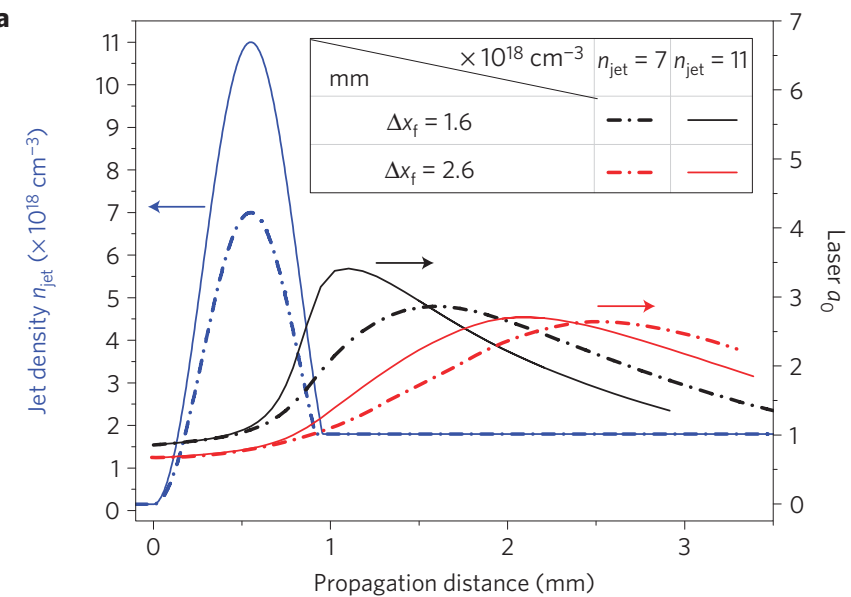

b

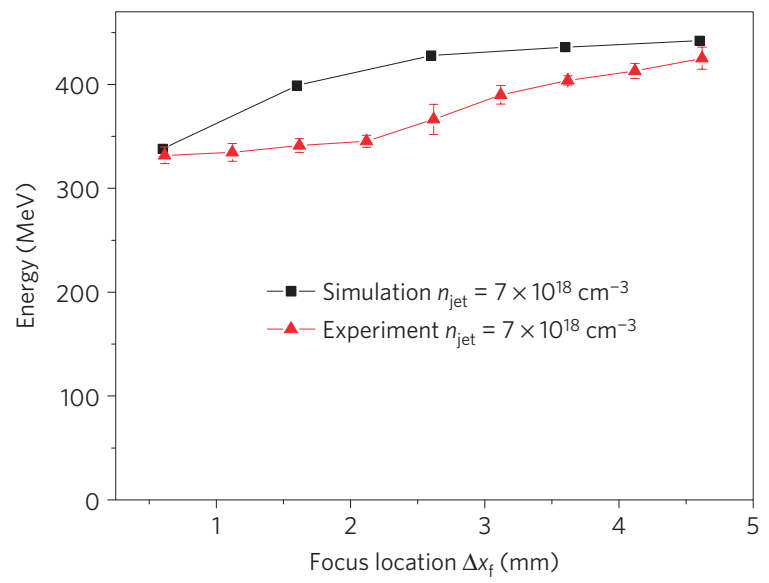

Figure 4 | Comparison of the energy gain observed in simulation and experiment, demonstrating tunability of electron-beam energy through control over laser pulse diffraction. a, Simulated evolution of the normalized vector potential $a_{0}$ as a function of propagation distance for different focal locations, $\Delta x_{f}=1.6 \mathrm{~mm}$ (black) and $\Delta x_{f}=2.6 \mathrm{~mm}$ (red), and peak densities, $n_{\text {jet }}=7 \times 10^{18} \mathrm{~cm}^{-3}$ (dash-dot lines) and $n_{\text {jet }}=11 \times 10^{18} \mathrm{~cm}^{-3}$ (solid lines). The blue lines show the longitudinal density profile. $\mathbf{b}$, Energy gain computed as a function of $n_{\text {jet }}$ for different focal locations, along with experimental data for the conditions of Fig. $2 b-g$. The error bars correspond to the standard deviation of the data.

capillary discharge waveguide ${ }^{2}$, where a higher density was used and diffraction mitigated to enable acceleration to dephasing and higher energy gain.

The shot-to-shot stability of the beams is significantly better than that achieved in previous experiments. The root mean square variation in energy, charge and angle for consecutive shots shown in Fig. $2 \mathrm{c}$ was $1.9 \%, 45 \%$ and $0.57 \mathrm{mrad}$, respectively, and as shown in Fig. $2 \mathrm{~b}-\mathrm{f}$ this high degree of stability is not limited to a narrow range of parameters. The charge stability was reduced to $6 \%$ by small changes to the density profile, representing more than a fourfold improvement over current state-of-the-art LPAs using the more complex injection scheme of multiple laser pulses ${ }^{28}$. The laser beam had a Strehl ratio of $\approx 0.9$ and the laser energy fluctuation was $3 \%$.

Deconvolving the laser energy fluctuation, which was correlated to charge fluctuation, indicates that the charge variation could be reduced to below $4 \%$. Injection assisted by partially ionized gas species could improve stability further because less self-focusing of the laser pulse is required. This could also be advantageous for matched guiding in the lower-density structure because the focused spot size could be larger.

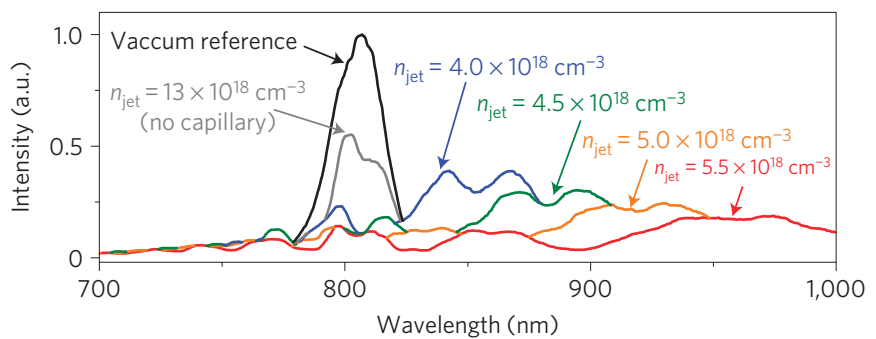

Figure 5 | Laser spectra for no plasma (black), jet only with density $13 \times 10^{18} \mathrm{~cm}^{-3}$ and length (FWHM) $0.75 \mathrm{~mm}$ (grey) and jet and capillary with jet length $0.55 \mathrm{~mm}$ and density between $4 \times 10^{18}$ and $5.5 \times 10^{18} \mathrm{~cm}^{-3}$ (coloured plots), each with $n_{\text {cap }} \approx 1.5 \times 10^{18} \mathrm{~cm}^{-3}$.

\section{Methods}

Laser. Pulses from the LOASIS Ti:sapphire laser system were focused onto the gas targets by a 2 -m-focal-length off-axis parabolic mirror to a focal spot size of $r_{0}=22 \mu \mathrm{m}$ (Fig. 1 ; ref. 2). This corresponds to a Rayleigh range $Z_{\mathrm{R}}=1.9 \mathrm{~mm}$. For these experiments the laser energy on target was $1.4 \mathrm{~J}$, corresponding to a peak intensity of $4 \times 10^{18} \mathrm{~W} \mathrm{~cm}^{-2}$ for a pulse length of $38 \mathrm{fs}$.

Laser and electron-beam diagnostics. Laser radiation emerging from the capillary passed through the electron spectrometer and was attenuated by reflection off two optically flat wedges. The energy of each laser pulse input to the waveguide was determined by a photodiode that was calibrated to the energy on target. The energy of pulses transmitted through the capillary was measured by loosely focusing the portion of the laser beam transmitted through the second wedge onto a pyroelectric energy meter that was cross-calibrated to the input photodiode.

The energy of electron bunches emerging from the plasma was measured by a magnetic electron spectrometer ${ }^{29}$. A $1.2 \mathrm{~T}$ magnet deflected the electrons onto phosphor screens imaged by four synchronously triggered CCD (charge-coupled device) cameras, enabling single-shot detection of electrons with energies in the range $0.01-0.14 \mathrm{GeV}$ and $0.17-1.1 \mathrm{GeV}$. Charge was obtained from the phosphor screens, which were cross-calibrated against an integrating current transformer. This diagnostic provided charge-density images of the electron beam with energy in one direction, and angle in the undispersed plane.

Capillary waveguide and embedded gas jet. The capillary employed had diameter $220 \mu \mathrm{m}$ and length $33 \mathrm{~mm}$. Hydrogen gas was flowed into the capillary through slots located $3.85 \mathrm{~mm}$ from each end of the capillary. Helium gas filled the injector nozzle, which was located at a distance of $1.38 \mathrm{~mm}$ after the entrance of the capillary. The discharge current was approximately sinusoidal, with peak current $250 \mathrm{~A}$. Data were taken for the laser arrival $390 \mathrm{~ns}$ after the onset of the discharge, which was within a few tens of nanoseconds from the peak of the discharge. The on-axis electron density and matched spot of the waveguide were calculated from the backing pressure as described in ref. 26. The matched spot of the waveguide was between 45 and $50 \mu \mathrm{m}$.

The density profile for the jet injector was measured with a Michelson interferometer with the probe beam shown in Fig. 1c. The injector experiments employed different gases (hydrogen, helium and a gas mix of $1 \%$ nitrogen balance helium), at plasma densities between $4 \times 10^{18}$ and $14 \times 10^{18} \mathrm{~cm}^{-3}$. The plasma length (FWHM) was scanned between $0.55 \mathrm{~mm}$ and $1.3 \mathrm{~mm}$, and the focal location from $3 \mathrm{~mm}$ before to $4 \mathrm{~mm}$ after the jet centre.

Transverse optical access to the jet was not available for the jet embedded into the capillary. To obtain the density in this case neutral gas interferometry with a wavefront sensor was carried out on a similar gas jet without capillary and found to be within $20 \%$ of simulations done using a commercial fluid dynamics code. Simulation of the jet embedded in the capillary showed the density profile not to be significantly affected and gas did not flow into the capillary section. The peak density in the simulation was $n_{\text {jet }}=13 \times 10^{18} \mathrm{~cm}^{-3}$ for the conditions of Fig. $2 \mathrm{a}$, but this is an upper limit, because after the discharge is struck Ohmic heating causes expansion of the gas and a reduction in density. An estimate of the decrease in density due to Ohmic heating was obtained by comparing laser-energy-depletion data of the jet alone with the jet embedded in the capillary. Energy depletion for jet and capillary should be equal to or greater than that for the jet alone for the same jet density. With this assumption, an upper limit to the density is $n_{\mathrm{jet}}=1 \times 10^{19} \mathrm{~cm}^{-3}$. By lowering the capillary density, energy depletion decreased. By assuming that the minimum observed energy depletion is close to the value of the jet alone, a better estimate for the conditions of Fig. $2 \mathrm{a}$ is $n_{\mathrm{jet}} \approx 7 \times 10^{18} \mathrm{~cm}^{-3}$. The initial gas density was halved, and this correction was applied for all data. Shot-to-shot stability density from the jet was measured to be at least as low as $4 \%$, which was the measurement error of the interferometric technique.

INF\&RNO. Calculations of the laser-plasma interaction (laser and wakefield evolution, energy gain for electrons) are carried out with the computational 
framework INF\&RNo (Integrated Fluid and Particle Simulation Code). INF\&RNO is a two-dimensional cylindrical $(r-z)$ code that adopts an envelope model for the laser pulse and makes use of the ponderomotive-force approximation to describe the interaction of the laser pulse with the plasma ${ }^{1}$. The plasma can be modelled using either a fluid or a fully kinetic description, the latter being realized through the particle-in-cell (PIC) approach. Both PIC and fluid modalities are integrated in the same framework, allowing for staged simulations (for example, PIC mode for injection and fluid mode for acceleration). The electromagnetic field associated with the plasma wake and the laser envelope are managed using a finite-difference approach. All the fields are discretized onto a grid and spatial derivatives in the evolution equations are computed with second-order upwind (in the longitudinal direction) or centred (in the transverse direction) finite-difference schemes. Temporal update of the wakefield is carried out using a second- or fourth-order Runge-Kutta integrator, whereas a second-order Crank-Nicolson method is adopted for the laser envelope. For the plasma description in the fluid modality, additional fields for the density and momentum density are included. In the PIC modality the plasma phase-space distribution is represented using numerical particles which are advanced solving the relativistic equations of motion with a fourth-order Runge-Kutta integrator. Quadratic shape functions are used for force interpolation and charge/current deposition onto the grid. Non-uniform/adaptive particle sampling is adopted to minimize statistical noise and increase accuracy within the dynamically interesting zones without greatly increasing the overall number of simulated particles. A boosted-Lorentz-frame modelling capability for the fluid modality is also available, allowing for a significant speed-up in the calculations. Compared with standard simulation tools, INF\&RNO allows for a reduction of many orders of magnitude in computational time, while retaining physical fidelity.

Received 22 December 2010; accepted 26 July 2011; published online 21 August 2011

\section{References}

1. Esarey, E., Schroeder, C. B. \& Leemans, W. P. Physics of laser-driven plasma-based electron accelerators. Rev. Mod. Phys. 81, 1229-1285 (2009).

2. Leemans, W. P. et al. GeV electron beams from a centimetre-scale accelerator. Nature Phys. 2, 696-699 (2006).

3. Leemans, W. P. et al. Observation of terahertz emission from a laser-plasma accelerated electron bunch crossing a plasma-vacuum boundary. Phys. Rev. Lett. 91, 074802 (2003).

4. Rousse, A. et al. Production of a keV x-ray beam from synchrotron radiation in relativistic laser-plasma interaction. Phys. Rev. Lett. 93, 135005 (2004).

5. Fuchs, M. et al. Laser-driven soft-x-ray undulator source. Nature Phys. 5, 826-829 (2009).

6. Leemans, W. P. \& Esarey, E. Laser-driven plasma-wave electron accelerators. Phys. Today 62, 44-49 (March, 2009).

7. Schroeder, C. B., Esarey, E., Geddes, C. G. R., Benedetti, C. \& Leemans, W. P. Physics considerations for laser-plasma linear colliders. Phys. Rev. ST Accel. Beams 13, 101301 (2010).

8. Mangles, S. P. D. et al. Monoenergetic beams of relativistic electrons from intense laser-plasma interactions. Nature 431, 535-538 (2004).

9. Geddes, C. G. R. et al. High-quality electron beams from a laser wakefield accelerator using plasma-channel guiding. Nature 431, 538-541 (2004).

10. Faure, J. et al. A laser-plasma accelerator producing monoenergetic electron beams. Nature 431, 541-544 (2004).

11. Umstadter, D., Kim, J. K. \& Dodd, E. Laser injection of ultrashort electron pulses into wakefield plasma waves. Phys. Rev. Lett. 76, 2073-2076 (1996).

12. Esarey, E., Hubbard, R. F., Leemans, W. P., Ting, A. \& Sprangle, P. Electron injection into plasma wake fields by colliding laser pulses. Phys. Rev. Lett. 79, 2682-2685 (1997).

13. Ting, A., Kaganovich, D., Gordon, D. F., Hubbard, R. F. \& Sprangle, P. Generation and measurements of high energy injection electrons from the high density laser ionization and ponderomotive acceleration. Phys. Plasmas 12, 010701 (2005).

14. Faure, J. et al. Controlled injection and acceleration of electrons in plasma wakefields by colliding laser pulses. Nature 444, 737-739 (2006).
15. Chen, M., Sheng, Z. M., Ma, Y. Y. \& Zhang, J. Electron injection and trapping in a laser wakefield by field ionization to high-charge states of gases. J. Appl. Phys. 99, 056109 (2006).

16. Pak, A. et al. Injection and trapping of tunnel-ionized electrons into laser-produced wakes. Phys. Rev. Lett. 104, 025003 (2010).

17. McGuffey, C. et al. Ionization induced trapping in a laser wakefield accelerator. Phys. Rev. Lett. 104, 025004 (2010).

18. Bulanov, S., Naumova, N., Pegoraro, F. \& Sakai, J. Particle injection into the wave acceleration phase due to nonlinear wake wave breaking. Phys. Rev. E 58, R5257-R5260 (1998)

19. Geddes, C. G. R. et al. Plasma-density-gradient injection of low absolute-momentum-spread electron bunches. Phys. Rev. Lett. 100, 215004 (2008).

20. Schmid, K. et al. Density-transition based electron injector for laser driven wakefield accelerators. Phys. Rev. ST Accel. Beams 13, 091301 (2010).

21. Faure, J., Rechatin, C., Lundh, O., Ammoura, L. \& Malka, V. Injection and acceleration of quasimonoenergetic relativistic electron beams using density gradients at the edges of a plasma channel. Phys. Plasmas 17, 083107 (2010).

22. Reitsma, A. J. W. et al. Simulation of electron postacceleration in a two-stage laser wakefield accelerator. Phys. Rev. ST Accel. Beams 5, 051301 (2002).

23. Tomassini, P. et al. Production of high-quality electron beams in numerical experiments of laser wakefield acceleration with longitudinal wave breaking. Phys. Rev. ST Accel. Beams 6, 121301 (2003).

24. Brantov, A. V. et al. Controlled electron injection into the wake wave using plasma density inhomogeneity. Phys. Plasmas 15, 073111 (2008).

25. Spence, D. J. \& Hooker, S. M. Investigation of a hydrogen plasma waveguide. Phys. Rev. E 63, 015401 (2001).

26. Gonsalves, A. J., Rowlands-Rees, T. P., Broks, B. H. P., van der Mullen, J. J. A. M. \& Hooker, S. M. Transverse interferometry of a hydrogen-filled capillary discharge waveguide. Phys. Rev. Lett. 98, 025002 (2007).

27. Rowlands-Rees, T. P. et al. Laser-driven acceleration of electrons in a partially ionized plasma channel. Phys. Rev. Lett. 100, 105005 (2008).

28. Rechatin, C. et al. Quasi-monoenergetic electron beams produced by colliding cross-polarized laser pulses in underdense plasmas. New J. Phys. 11, 013011 (2009).

29. Nakamura, K. et al. Broadband single-shot electron spectrometer for GeV-class laser-plasma-based accelerators. Rev. Sci. Instrum. 79, 053301 (2008)

\section{Acknowledgements}

The authors would like to thank E. Cormier-Michel, M. Chen, J. Mefford, N. Matlis and G. Plateau for discussions. We appreciate contributions from D. Syversrud, Z. Eisentraut, K. Sihler and N. Ybarrolaza. This work was supported by the Director, Office of Science, Office of High Energy Physics, US Department of Energy, under contract No DE-AC02-05CH11231, by the National Science Foundation under grants PHY-0935197 and PHY-0917687 and by the Defense Advanced Research Projects Agency (DARPA).

\section{Author contributions}

A.J.G. worked on the design and execution of the experiments, analysed the data and was the primary author of the paper. W.P.L. conceived of and supervised the project. C.L. fabricated the targets and carried out the neutral gas fluid simulations. K.N. analysed electron-beam images. C.B. carried out the simulations of the laser-plasma interactions. E.E., C.G.R.G., C.B. and C.B.S. contributed to the understanding of experimental data, including the formulation of the theory of phase velocity control through self-focusing. C.T. worked on laser systems. The team for the experiments employing the jet embedded into the capillary included K.N., C.L., D.P. and A.J.G.. The experimental team for the gas jet experiments included K.N., C.L., T.S., J.O., J.V.T., S.S. and A.J.G.

\section{Additional information}

The authors declare no competing financial interests. Reprints and permissions information is available online at http://www.nature.com/reprints. Correspondence and requests for materials should be addressed to W.P.L. 